\title{
OPTIMIZACIÓN DE LA AUTOFORMACIÓN A TRAVÉS DE LA ENSEÑANZA VIRTUAL
}

\author{
Self-learning Optimization through Virtual Teaching
}

\section{Otimização da auto formação através do ensino virtual}

\section{RESUMEN}

En la última década, las instituciones superiores educativas han sufrido una gran transformación, manifiesta en la tendencia hacia la globalización, generalización y expansión del conocimiento. Un escenario educativo innovador, donde el acceso a la formación es un fenómeno generalizado. Los profesionales deben ser capaces de mantener sus experiencias actualizadas a medida que se producen continuos avances en sus áreas de trabajo. Es imprescindible el desarrollo de un entorno educativo innovador, que permita poner a disposición de profesores y estudiantes herramientas que ofrezcan la posibilidad de utilizar nuevos métodos de enseñanza a través de las tecnologías de la información y la comunicación (TIC). En el trabajo que aquí presentamos, se analiza un método de enseñanza-aprendizaje más activo y participativo. El presente proyecto permite al alumno disponer de un conjunto de recursos, que favorecen la autoevaluación y autoformación, a la vez que facilita su trabajo personal y en equipo.

PALABRAS CLAVE: 1: enseñanza reflexiva, innovación docente, plataforma Blackboard, autoevaluación, e-learning.

\section{EVALUADO:22 DE FEBRERO DE 2016}

Antonio Martín Montes (España) Universidad de Sevilla

Doctor en Inteligencia de Sistemas antoniomartin@dte.us.es

\section{en}

\section{ABSTRACT}

During the last decade, higher education institutions have suffered a big change displayed in the globalization, generalization, and expansion of knowledge. It shows an innovative educational scenario where the access to training is a general phenomenon. Professionals should be able to update their experiences at the same time that continuous progress within their work areas is produced. It is mandatory to develop an innovative educational environment that provides teachers and students with the tools that enable the use of new teaching methods through information and communication technologies (ICTs). This project analyzes a more active and participative teaching-learning method, where the student can use a set of resources that enables his/her self-evaluation and self-formation while supporting individual and team work

KEYWORDS:1Reflexive teaching, teaching innovation, Blackboard platform, self-evaluation, e-learning.

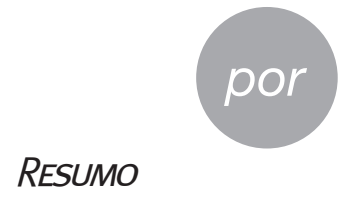

$\mathrm{Na}$ última década, as instituições superiores educativas têm sofrido uma grande transformação, manifesta na tendência para a globalização, generalização e expansão do conhecimento. Um cenário educativo inovador, onde o acesso à formação é um fenômeno generalizado. Os profissionais devem ser capazes de manter as suas experiências atualizadas na medida em que se produzem contínuos avanços nas suas áreas de trabalho. É imprescindível o desenvolvimento de um entorno educativo inovador, que permita pôr a disposição de professores e estudantes ferramentas que ofereçam a possibilidade de utilizar novos métodos de ensino através das tecnologias da informação e a comunicação (TIC). No trabalho que aqui apresentamos, analisa-se um método de ensino-aprendizado mais ativo e participativo. $\mathrm{O}$ presente projeto permite ao aluno dispor de um conjunto de recursos, que favoreça a auto avaliação e auto formação, à vez que facilita o seu trabalho pessoal e em equipe.

PALAVRAS CHAVE: ensino reflexivo, inovação docente, plataforma Blackboard, auto avaliação, e-learning. 


\section{INTRODUCCIÓN}

El concepto de docencia universitaria tradicional se ha visto modificado por los principios definidos en el Espacio Europeo de Educación Superior. Un cambio metodológico que motiva el concepto de autoevaluación y promueve todas aquellas actividades académicas que faciliten el autoaprendizaje. En este escenario, es fundamental que los estudiantes adquieran nuevos hábitos autoformativos. En esta nueva realidad, el autoaprendizaje se convierte en un requisito indispensable. Formar a los futuros profesionales con capacidades de autoformación es un objetivo transversal en los actuales currículos de cualquier grado universitario (Johnson y Hornik, 2008). Es necesario construir un nuevo marco educativo, utilizando para ello estrategias y técnicas para que el aprendizaje se produzca de la forma más asequible, práctica, contextualizada y funcional posible. Un nuevo esquema que no consista solo en la mera transmisión de conocimientos y procedimientos, sino que, además, sirva para adquirir nuevas habilidades, capacidades y aptitudes mediante el autoaprendizaje.

En este estudio, se cubre de forma completa la autoformación y autoevaluación de los alumnos de Ingeniería, utilizando para ello una plataforma virtual, como es Blackborad, junto con herramientas de elaboración de contenidos, generación de encuestas, etc. Aunque existen diferentes trabajos y propuestas realizadas en esta línea (Foroughi, 2005; Lin, 2011; Lu y Zhao, 2008; Maiorana, Sgarbossa y Salomoni, 2008; Ochoa et al., 2003), el estudio que aquí presentamos cubre facetas y aspectos no recogidos de manera completa en estos trabajos. La idea de este proyecto se centra en la preparación de material didáctico relacionado con cada una las actividades de laboratorio de la asigna-
Panorama I pp. 41-49। Volumen 9 । Julio-diciembre | 2015 I 42 Número 17 | tura de Sistemas de Información Industrial, del Máster Universitario en Sistemas Inteligentes en Energía y Transporte impartido por la Escuela Politécnica Superior de la Universidad de Sevilla. Se realiza una propuesta de cambio en la forma de enseñar, para que esta pase a ser más activa y participativa (Carstensen y Bernhard, 2007). Así se ha elaborado un material didáctico original de apoyo para dicha asignatura. En concreto, se ha completado el desarrollado de un sistema de autoaprendizaje y entrenamiento interactivo a través

de la plataforma de enseñanza virtual WebCT de la Universidad, que permitirá resolver cuestiones sobre las prácticas y suplir así las posibles carencias que presentan los alumnos en este aspecto (Salovaara, 2005).

La estructura del trabajo es la siguiente: en primer lugar, se presentan las razones que nos han llevado a la realización de este proyecto de innovación. En la sección siguiente, presentamos los objetivos perseguidos en nuestro proyecto y la metodología desarrollada. A continuación, se presenta un resumen de nuestra propuesta, señalando sus elementos principales. En la sección siguiente, se realiza un análisis sobre esta nueva sistemática y método de evaluación y se muestran los resultados obtenidos. Por último, se presentan las conclusiones y las posibles líneas futuras de investigación y mejora.

\section{OBJETIVO Y PERSPECTIVA DE LA PROPUESTA}

Los procedimientos docentes utilizados para la formación de los alumnos en las carreras de Ingeniería relacionados con la electrónica deben ser capaces de aunar conceptos básicos teóricos con un sentido práctico y realista que permita la plasmación de ideas en objetos reales. El rol del profesor no debe limitarse únicamente a observar el trabajo de los grupos, sino que debe supervisar activamente el proceso de construcción y transformación del conocimiento, así como las interacciones de los miembros de los distintos grupos (Cabero, 2006).

La asignatura de Sistemas de Información Industrial se imparte en el Máster Universitario en Sistemas Inteligentes en Energía y Transporte por la Universidad de Sevilla. Tiene carácter troncal y un total de seis créditos. Tres de estos créditos son de carácter práctico, dentro de los cuales estarían los trabajos de laboratorio. El alumno realiza cinco actividades de dos horas de duración a lo largo del curso académico.

Esta organización permite optimizar y maximizar el aprendizaje, ya que los alumnos trabajan juntos en la consecución de las diferentes tareas que el profesor asigna en cada una de las actividades. Cada práctica incluye el montaje, el ajuste/prueba y la evaluación de los subsistemas de medidas, así como realizar las 
diferentes operaciones y los experimentos propuestos en los enunciados. Tras la realización de las actividades de aprendizaje en el laboratorio, los alumnos elaboran una memoria descriptiva del trabajo realizado, que recogerá, además, el análisis de los resultados obtenidos. En estas prácticas, el enfoque instruccional está centrado en el estudiante, utilizando para ello pequeños grupos de trabajo, en general, formado por dos personas. Cada grupo va rotando por los puestos del laboratorio donde está dispuesto el instrumental de laboratorio, el material de montaje y las aplicaciones y el equipamiento informático necesario para la realización de las actividades.

\section{MOTIVACIÓN DOCENTE}

La experiencia docente efectuada se ha hecho desde el convencimiento de que el proceso de aprendizaje al que nos enfrentamos en el nuevo Espacio Europeo de Educación Superior no se reduce a la mera transmisión de información. Este nuevo espacio va aún más lejos, en el sentido de generar destrezas que enriquezcan al alumno ante su futuro profesional (Zawojewski, Diefes-Dux y Bowman, 2008), así como fomentar actitudes que debe tener todo universitario hacia el conocimiento en general y hacia el conocimiento científico en particular. Esto implica necesariamente plantear la renovación de las técnicas docentes, así como la incorporación de las tecnologías de la información y de la comunicación (TIC) y el uso de herramientas software en el proceso de enseñanza-aprendizaje del alumno (Case y Light, 2011).

La meta principal del trabajo que presentamos es flexibilizar el proceso de aprendizaje de la asignatura y paliar algunos de los problemas detectados en los últimos años, en la que existe un elevado porcentaje de alumnos que encuentran dificultades durante el desarrollo de las diferentes actividades. En primer lugar, la pérdida de tiempo al inicio de cada práctica, debido a la falta de preparación previa de su contenido. Aunque los enunciados de cada una de las prácticas están disponibles desde el comienzo de curso, la mayor parte del alumnado llega al laboratorio sin haberlos leído previamente. Para evitarlo, se establece un sistema de evaluación que incentive al alumno a llevar la asignatura al día. Otra dificultad detectada está relacionada con la realización de las memorias, que contienen el resumen del trabajo efectuado en el laboratorio y el análisis de resultados. La calidad de estas memorias está bajando considerablemente, presentando, además, clara tendencia a la copia entre compañeros.

Para el despliegue de nuestra propuesta, nos hemos basado en la plataforma de enseñanza virtual de nuestra Universidad. Todo el material didáctico elaborado se pone a disposición de los alumnos mediante el espacio docente que a tal efecto la asignatura de Sistemas de Información Industrial tiene disponible en dicha plataforma (figura 1).

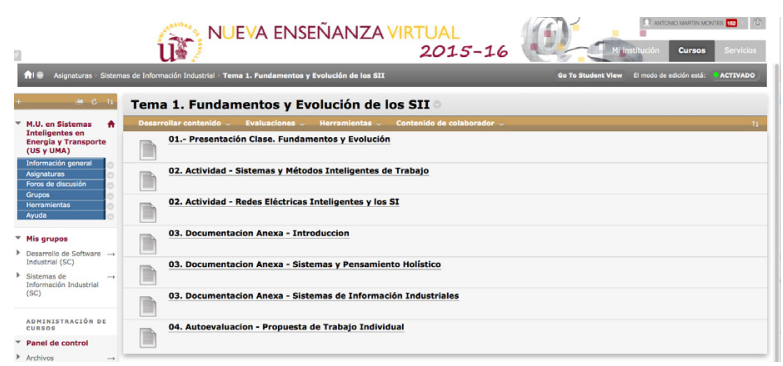

Figura 1. Contenido de la asignatura Blackboard.

La plataforma de enseñanza virtual Blackboard pone a disposición de toda la comunidad universitaria un conjunto de utilidades para la docencia a través de internet, que permite complementar la enseñanza presencial, y que facilita, además, la educación a distancia. Representa una gran interactividad y sencillez de uso, que simplifica la tarea de comunicación entre profesores y alumnos. Asimismo, Blackboard ofrece al profesor un conjunto de herramientas para la monitorización de los estudiantes, creación de exámenes, facilidades para el control del acceso al curso, asignación de claves, transferencias de ficheros, etc. Dicha plataforma es gestionada por el Servicio de Informática y Comunicaciones (SIC) de la Universidad, que se complementa con un conjunto de servicios de apoyo pedagógico a la elaboración de contenidos y un plan de formación del profesorado. Las razones que justifican el uso de esta plataforma en nuestro trabajo ha sido principalmente la experiencia adquirida por el profesorado en otros proyectos anteriores de innovación.
Optimización de la autoformación a través de la enseñanza virtual 


\section{ENFOQUE Y FLUJO METODOLÓGICO}

Para conseguir los objetivos señalados, y contribuir, Antonio Martín Montes | además, a la consecución de los objetivos definidos en la programación de la asignatura, hemos cambiado la sistemática de esta. Se ha elaborado un material didáctico mediante una nueva metodología que proporciona al alumno una ayuda en el proceso de adquisición de los conocimientos prácticos previos y posteriores a la realización del trabajo en el laboratorio. El profesor deja de ser la fuente de todo conocimiento y pasa a actuar como guía de los alumnos, facilitándoles el uso de los recursos y las herramientas que necesitan para modelar nuevos conocimientos y destrezas. La puesta en operación del proyecto plantea exigencias tanto a profesores como a estudiantes. Al profesor se le exige principalmente un trabajo planificado con mucha antelación, así como la preparación de una cantidad y diversidad mayor de materiales e instrumentos educativos. Por parte del estudiante, pone en sus manos la responsabilidad de su propio aprendizaje, al tener la garantía de una disponibilidad de la plataforma web las 24 horas del día.

El diseño del material docente se ha realizado de acuerdo con las normas de accesibilidad actuales y siguiendo directrices consensuadas por todos los profesores de la asignatura. La metodología desarrollada en ella se ha basado en estrategias propias de una enseñanza autónoma y, por supuesto, activa (Cabero y Morales, 2004). Centrada en la figura del alumno como elemento clave del sistema de formación y con una participación del profesor/tutor como dinamizador del proceso de aprendizaje. El procedimiento considera tres dimensiones esenciales de la actividad docente: enseñanzas teóricas, actividades prácticas y trabajo personal del alumno. Estas dimensiones se suceden cronológicamente en el

Panorama I pp. 41-49 I Volumen 9 I Número 17 |

La figura 2 muestra el flujo de trabajo en el proceso de formación de la asignatura. En los siguientes apartados, se procede al estudio en detalle de cada uno de ellos.

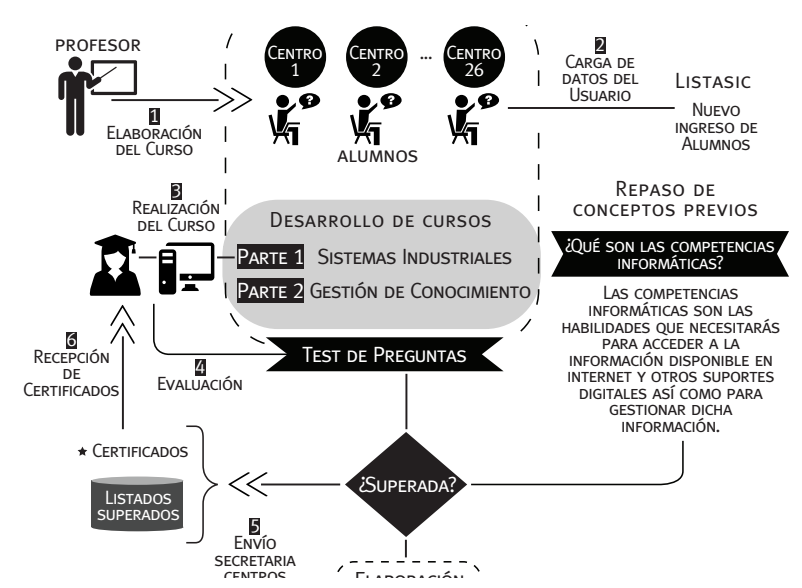

Figura 2. Diagrama de flujo de la metodología seguida.

EVALUACIÓN INICLAL DE LOS CONOCIMIENTOS TEÓRICOS

El método de trabajo propuesto permite adaptar la enseñanza de la asignatura a los diferentes ritmos de aprendizaje de los alumnos (Brockman y McGill, 2001), siempre dentro de unos límites de plazo establecidos, y siguiendo la programación y secuencia de las materias que se han ido superando. Con el fin de posibilitar el proceso de autoevaluación, existe un ejercicio inicial que capacita al alumno para la comprobación de los conocimientos teóricos sobre diferentes aspectos de la práctica por realizar. La utilización de herramientas de autoevaluación del conocimiento va a optimizar el proceso de aprendizaje de los alumnos en todos los conceptos teóricos. En este trabajo, la autoevaluación es empleada como un instrumento para que el alumno conozca y tome conciencia de cuál es su progreso individual en el proceso de enseñanza-aprendizaje. Con ello, se incentiva al alumno a preparar de forma previa el contenido de la práctica, lo cual va a mejorar el posterior desarrollo y aprovechamiento de las actividades realizadas en el laboratorio. Esta prueba se organiza como un banco de preguntas, que promueve la reflexión sobre la práctica y el propio proceso de aprendizaje (figura 3 ).

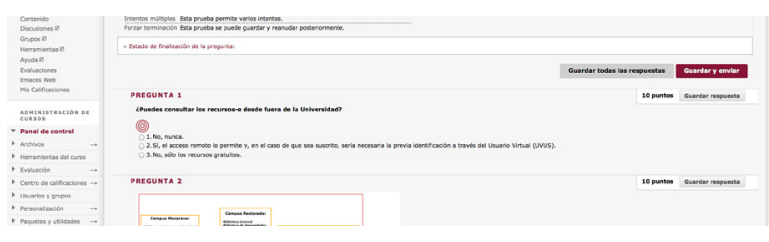

Figura 3. Módulo de evaluación final. 
Para continuar con el desarrollo de la práctica, el alumno tiene que superar satisfactoriamente este primer cuestionario. Para ello, tiene que responder correctamente $50 \%$ de las cuestiones iniciales, teniendo únicamente tres intentos para superarlo. En la figura 4, se pueden observar los resultados obtenidos tras el proceso de autoevaluación.

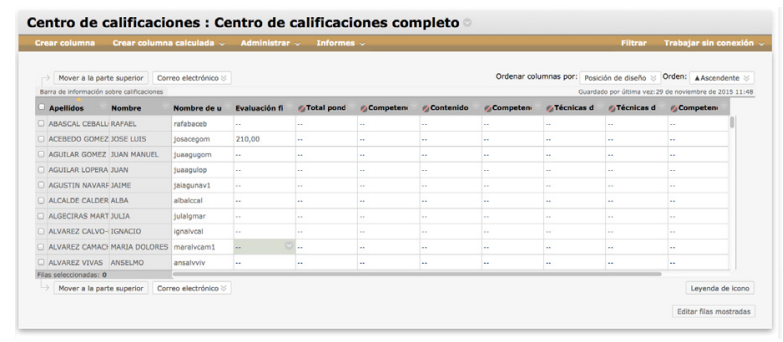

Figura 4. Módulo de evaluación final.

\section{DESARROLLO DE ACTIVIDADES EN EL LABORATORIO}

Una vez superada la autoevaluación inicial, el alumno comienza el módulo de aprendizaje de la práctica y las actividades que se proponen. El enfoque de nuestra asignatura es eminentemente práctico, y resalta la comprensión y aplicación de resultados experimentales. La metodología que se ha seguido aumenta el interés del alumno mediante la realización de actividades guiadas en el laboratorio y una adecuada selección de ejemplos prácticos (Exley y Dennick, 2007). Asimismo, la plataforma incorpora diferentes fuentes del conocimiento, que ponen al alcance de los participantes apuntes y documentación complementaria para su descarga, así como la identificación de sitios web de consulta donde poder completar la información transmitida en las clases (Rosenberg, 2001). La Blackboard también contiene enlaces y vínculos webs especializados en aspectos fundamentales y destacables de la asignatura.

Los múltiples apartados que componen la unidad docente están reflejados en una serie de módulos de aprendizaje, que definen el contenido didáctico, las diversas tareas y operaciones que el alumno llevará a cabo en cada una de las prácticas. La información se presenta estructurada para hacer más fácil la elección de los temas y permitir el itinerario de desarrollo (Arenas et al., 2009). Toda la información se almacena de manera ordenada y organizada, el acceso a ella se efectúa de forma automática y los procesos de búsqueda tienen costes reducidos. Asimismo, el material incluye resultados teóricos y demostraciones matemáticas sencillas o intuitivas que puedan ayudar a formar al alumno en los aspectos teóricos necesarios (figura 5).

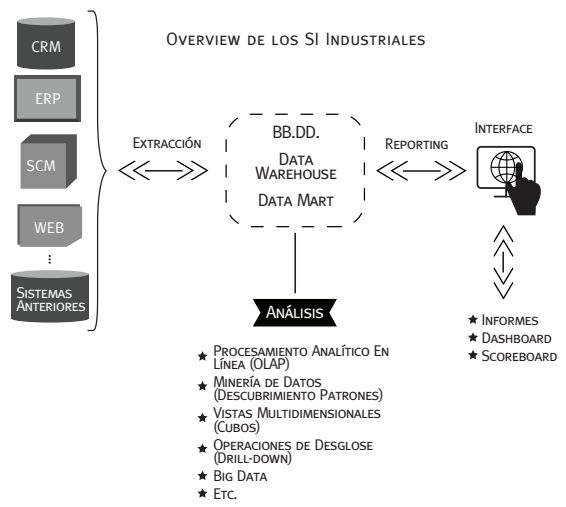

Figura 5. Módulo de aprendizaje de sistemas industriales.

La plataforma Blackboard ofrece diferentes utilidades que facilitan nuevas vías de comunicación entre profesor/alumno, y que amplían las posibilidades de aprendizaje: correo electrónico, chat, foro, pizarra electrónica, bases de datos de imágenes, glosarios, tareas, sistema de anotación de páginas, calificaciones accesibles en línea y calendario, entre otras. Para la generación de los contenidos interactivos compatible con la plataforma Blackboard, se ha utilizado la herramienta Wimba Create (WimbaCreate, 2012). Wimba Create se integrada como una barra de menú nueva dentro de Microsoft Word 2003 y 2007, y posibilita de forma muy sencilla generar contenidos para e-learning en numerosos formatos. Este software permite que materiales escritos y desarrollados en formato Word (.doc, .docx) sean convertidos de modo fácil y rápido en un curso en línea, tras lo cual genera un conjunto de páginas web, que incluye funciones interactivas y enlaces de navegación.

\section{EVALUACIÓN FINAL Y SISTEMA DE ENCUESTA}

Tras haber acometido la realización de todas las actividades de la práctica, existe una sección que contiene una serie de campos de entrada de texto. En este apartado, el alumno debe reflejar todos aquellos aspectos que tienen que ver con el desarrollo de la práctica en el laboratorio y que sirven como memoria final (Suárez, 2010). La información aportada por el alumno sirve, además, para
Optimización de la autoformación a través de la enseñanza virtual 
Antonio Martín

Montes I
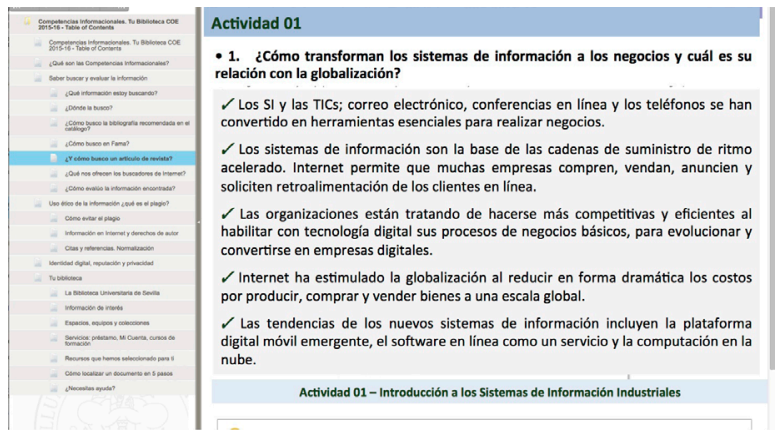

Figura 6. Campo de entrada, resumen de la práctica.

A la finalización de cada práctica de laboratorio, y con el fin de evaluar diferentes aspectos relacionados con la competencia de los alumnos y su evolución en el trabajo en grupo, se establece un formulario de encuesta. La encuesta docente permite recoger la satisfacción de los estudiantes sobre la actividad desarrollada en el laboratorio, que permite a los profesores de la asignatura conocer la opinión de los estudiantes. Contiene doce preguntas, donde los alumnos completan la opinión sobre la sistemática y las operaciones realizadas. Las cuestiones planteadas indagan sobre tres dimensiones centrales del modelo pedagógico: metodología docente, valoración general de la formación y objetivos y contenidos (figura 8).

\section{D.PINA páginas, 12 preguntas}

Encuesta de satisfacción. Práctica de Laboratorio Fotodiodo - Instrum. Electrónica 2011/2012. 1.- Objetivos y contenidos

1.1.- Con esta práctica se han cumplido tus expectativas Requerida,

$\mathrm{O}_{1} \mathrm{O}_{2} \mathrm{O}_{3} \mathrm{O}_{4} \mathrm{O}_{5}$

1.2.- Los temas se han tratado con la claridad suficiente en la práctica Requerida,

$\bigcirc_{1} \bigcirc_{2} O_{3} \bigcirc_{4} \bigcirc_{5}$

1.3.- El tiempo dedicado al desarrollo de la práctica te ha parecido adecuado Requerida,

$0_{1} O_{2} O_{3} O_{4} O_{5}$

Figura 8. Encuesta docente.

Panorama I

Esta información permite a los profesores de la asignatura poder llevar a cabo una evaluación de la utilidad efectiva de uso, del material didáctico, con un doble objetivo: establecer las áreas de mejora y proporcionar un informe detallado sobre cada una de las prácticas, a partir de la cual pueda plantearse sus puntos fuertes. Estos aspectos posibilitan mejorar la eficacia de los materiales de estudio, de las interacciones alumnos-profesor y de la calidad de las prácticas en general.
Para la creación de dicha encuesta, se ha utilizado la plataforma Opina (Opina, 2012), desarrollada por nuestra Universidad, que permite modelar cuestionarios o encuestas de una forma eficiente y fácil. Esta aplicación posibilita realizar, desde cualquier punto con acceso web a nuestros cuestionarios, gestión de alumnos, establecer modificaciones, así como parametrizar y configurar todas las opciones de las que consta la encuesta.

Para las respuestas relacionadas con la valoración general de la formación, la encuesta contiene una escala de 1 a 5 , coincidentes con los valores siguientes: 1, muy en desacuerdo; 2 , en desacuerdo; 3 , ni de acuerdo ni en desacuerdo; 4, de acuerdo; y 5, muy de acuerdo. Para las respuestas relacionadas con la metodología docente, objetivos y contenidos, los valores asociados son: 1 , muy mala; 2 , mala; 3 , regular; 4, buena; y 5 , muy buena.

\section{RESULTADOS}

Una vez realizados todos los experimentos, y recabados los datos correspondientes, en esta sección procedemos al análisis de la información atendiendo a diferentes aspectos. Para la realización del estudio, inicialmente partíamos de un análisis básico en el que únicamente teníamos en cuenta la cantidad y la calidad del trabajo efectuado por cada miembro del grupo (Ballesteros y Cabero, 2010). Pronto detectamos que era necesario disponer de indicadores más precisos, que permitiesen obtener información más determinante, para extraer conclusiones. En este sentido, estamos evaluando entre las siguientes características: calidad del trabajo realizado por el alumno, cantidad habitualmente medida en número de horas, proporción de trabajo llevada a cabo por el alumno en relación con el esfuerzo total desarrollado por el grupo, puntualidad, cumplimiento y entrega de tareas completas, seguimiento y revisión, etc.

Otro aspecto importante en el análisis tiene que ver con los datos aportados por la encuesta de satisfacción, que tienen un gran valor. Los datos que hemos analizado han sido los recogidos mediante la encuesta, y suministrado por los alumnos de la asignatura en 2014-2015. Esta información sirve como una retroalimentación, necesaria para la mejora de la metodología y del contenido del proyecto (Caplan y Graham, 2011). La encuesta de satisfacción fue contestada por 96 alumnos de forma voluntaria. Como se evidencia en la figura 9, la mayoría de los alumnos valoró positivamente la experiencia, y considera que la formación recibida ha sido interesante. 
Respecto de la pregunta: ¿esta formación será útil para mi desarrollo profesional?, $50 \%$ está muy de acuerdo y de acuerdo $37 \%$, mientras que ni de acuerdo ni en desacuerdo $13 \%$. En cuanto a la valoración global de la formación, $50 \%$ de los alumnos señaló que fue muy buena, y muy buena $13 \%$, solo $12 \%$ indicó regular y $25 \%$ desacuerdo.

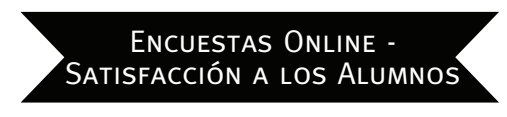

\section{LA METODOLOGÍA SEGUIDA ES ÚTIL PARA EL DESARROLLO PERSONAL Y PROFESIONAL}

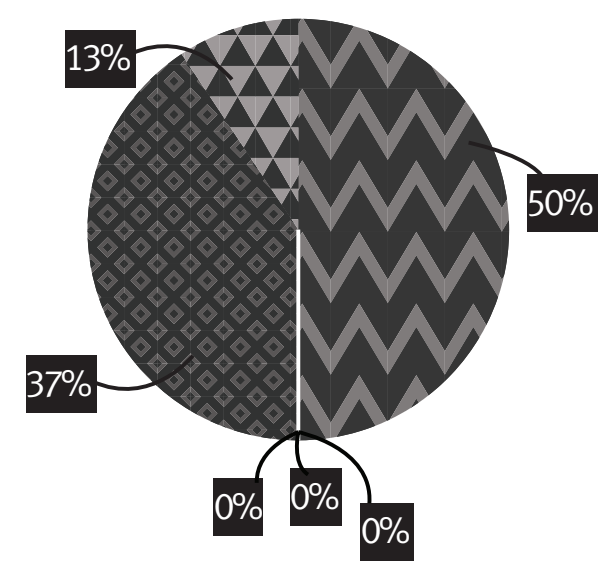

\section{LAS ACTIVIDADES REALIZADAS EN GENERAL TIENEN UNA BUENA VALORACIÓN}

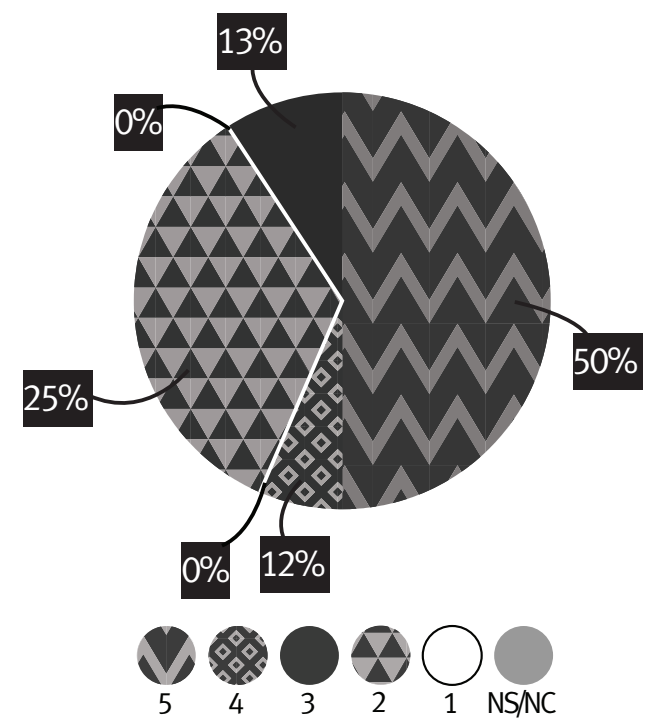

Figura 9. Resultados de la encuesta docente.

Teniendo en cuenta estos resultados, junto con las demás cuestiones presentadas en la encuesta y el test de autoevaluación inicial, podemos afirmar que la experiencia ha servido al alumno para fomentar el espíritu crítico, desde la perspectiva del aprendizaje y sobre la responsabilidad de uno mismo en su proceso formativo. Los alumnos describieron el ambiente en el que desarrollaron las actividades prácticas como cordial y participativo, asimismo valoraron la organización como buena.

\section{DISCUSIÓN}

La educación superior actual impone un proceso de renovación metodológica, que exige el diseño de nuevos métodos docentes que favorezcan el aprendizaje activo del estudiante, que lo enseñen a autoaprender y que integren la teoría con la práctica. Un contexto que favorezca la interacción profesor-alumno y la actuación del alumno como profesional. Este proyecto se ha basado en estrategias propias de una enseñanza activa y autónoma, centrada en la figura del alumno como elemento clave del sistema de formación y con una participación del profesor/tutor como dinamizador y facilitador del proceso de aprendizaje. $\mathrm{El}$ procedimiento seguido ha permitido al alumno realizar el análisis, la reflexión y el contraste sobre su propia actuación como profesional.

En la experiencia docente llevada a cabo, se pone de manifiesto que el material desarrollado es un instrumento útil para permitir al docente asumir la función de dinamizador del proceso de aprendizaje y favorecer la participación del alumno. El material elaborado ha sido esencial para que los alumnos adquieran conocimientos, habilidades y actitudes y, de este modo, desarrollar nuevas competencias. La metodología del proyecto motiva al alumno, promociona su autonomía y el pensamiento crítico y reflexivo.

El desarrollo de las acciones prácticas, y su posterior evaluación mediante la plataforma de enseñanza virtual, ha permitido al alumno alcanzar diferentes objetivos. Por un lado, ha posibilitado diversificar y flexibilizar la enseñanza tradicional, además de la estimación del grado de eficacia de los recursos, mediante un proceso de autoevaluación y encuesta. Para el alumnado, la experiencia fue evaluada positivamente, mostró una gran satisfacción al finalizar la elaboración de las actividades en el laboratorio y ha servido para profundizar en la materia, aprender a autoevaluarse y a utilizar las nuevas 
Antonio Martín

Montes I

permitido establecer criterios de evaluación y contenidos mínimos imprescindibles para superar la materia.

Para finalizar, se propone como trabajo futuro la integración de la plataforma Blackboard con otras plataformas de enseñanza virtual y repositorios de materiales didácticos, mejorar el contenido didáctico, agregar nuevas funcionalidades a la interfaz de comunicación con los estudiantes y perfeccionar el sistema de autoevaluación y análisis de resultados de la encuesta, entre otros.

\section{REFERENCIAS BIBLIOGRÁFICAS}

1. Arenas, F. J., Domingo, M.A., Molleda, G., Ríos, M. A. y Ruiz, J. C. (2009). Aprendizaje interactivo en la educación superior a través de sitios web: un estudio empírico. Pixel-Bit, Revista de Medios y Educación, 35, 127-145.

2. Ballesteros, C. y Cabero, J. (2010). Usos del e-learning en las universidades andaluzas: estado de la situación y análisis de buenas prácticas. Pixel-Bit, Revista de Medios y Educación, 3(37), 7-18.

3. Brockman, G. y McGill, H. (2001). El aprendizaje critico en la enseñanza superior. Madrid: Morata.

4. Cabero, J. (2006). Formación del profesorado universitario en estrategias metodológicas para la incorporación del aprendizaje en red en el espacio europeo de educación superior. Pixel-Bit, Revista de Medios y Educación, 27, 11-29.

5. Cabero, J.y Morales, J. A. (2004). La red como instrumento de formación: bases para el diseño de materiales didácticos. Pixel-Bit, Revista de Medios y Educación, 22, 5-23.

Panorama I

pp. $41-49$ I

Volumen 9 ।

Número 17 I

7. Carstensen, A. K. y Bernhard, J. (2007, junio). Critical Aspects for Learning in an Electric Circuit Theory Course-an Example of Applying Learning Theory and Design-based Educational Research in Developing Engineering Education. En First International Conference on Research in Engineering Education (pp. 22-24). Honolulu.
8. Case, J. M. y Light, G. (2011). Emerging Methodologies in Engineering Education. Journal of Engineering Education, 100(1), 186-210.

9. Exley, K. y Dennick, R. (2007). Enseñanza en pequeños grupos en educación superior. Madrid: Narcea.

10. Foroughi, R. (2005, octubre). Work in ProgressGraphical Tools for Self-Evaluation in E-Learning. En Frontiers in Education, 2005. FIE'05. Proceedings 35th Annual Conference (pp. F3G-F3G). IEEE.

11. Johnson, R. D. y Hornik, E. (2008). An Empirical Examination of Factors Contributing to the Creation of Successful E-Learning Environments. International Journal of HumanComputer Studies, 66(5), 356-369.

12. Lin, K. (2011). E-Learning Continuance Intention: Moderating Effects of User E-Learning Experience. Computers E Education, 56(2), 515-526.

13. Lu, W. y Zhao, C. (2008, diciembre). Design of Virtual Learning Community Applied to Further Education for Teachers. En Knowledge Acquisition and Modeling, 2008. KAM'08. International Symposium on (pp. 508-511). IEEE.

14. Maiorana, C., Sgarbossa, L. y Salomoni, V. (2008). New Methodologies in Teaching E-Structural Mechanics Using WWW. Computer Applications in Engineering Education, 16(3), 189-210.

15. Ochoa, S. F., Pino, J.A., Baloian, N. y Fuller, D. A. (2003). ICESEE: A Tool for Developing Engineering Courseware. Computer Applications in Engineering Education, 11(2), 53-66.

16. Opina: Gestor de encuestas v1.5.1 (r3230) Copyright (C) 2010 klicap - ingeniería del puzle. OPINA. Recuperado de http://portalapps.us.es/ opina/

17. Rosenberg, M.J. (2001). E-Learning: Strategies for Delivering Knowledge in the Digital Age. Nueva York: McGraw-Hill.

18. Salovaara, H. (2005). Achievement Goals and Cognitive Learning Strategies in Dynamic Contexts of Learning. Oulu, Finlandia: University of Oulu.

19. Sørensen, E. (2009). The Materiality of Learning: Technology and Knowledge in Educational Practice. Nueva York, NY: Cambridge University Press.

20. Suárez, C. (2010). Aprendizaje cooperativo e interacción asíncrona textual en contextos educativos virtuales. Pixel-Bit, Revista de Medios y Educación, 36, 53-67. 
21. WimbaCreate. Create Course Content.

Recuperado de http://www.wimba.com/ products/wimba_create

22. Zawojewski, J. S., Diefes-Dux, H. A. y

Bowmans, K. (2008). Models and Modeling in

Engineering Education: Designing Experiences

for all Students. Rotterdam, Países Bajos: Sense

Publishers. 